\title{
Development and Validation for the Simultaneous Quantification of Prazosin, Amlodipine, Diltiazem and Verapamil in API, Dosage Formulation and Human Serum by RP-HPLC: Application to in vitro Interaction Studies
}

Najma Sultana', Saeed Arayne $\mathrm{M}^{{ }^{*}}$ and Shabana Naz Shah ${ }^{1}$

${ }^{1}$ Research Institute of Pharmaceutical Sciences, Faculty of Pharmacy University of Karachi, Karachi-75270, Pakistan

2Department of Chemistry, University of Karachi, Karachi-75270, Pakistan

\section{Abstract}

A novel reversed-phase liquid chromatographic method with UV detection for rapid and accurate simultaneous quantitation of prazosin (PRZ) and the key calcium channel blockers (CCBS), amlodipine besylate (AML), diltiazem hydrochloride (DIL) and verapamil hydrochloride (VER) in active pharmaceutical ingredients, pharmaceutical dosage formulations and human serum has been developed and validated according to $\mathrm{ICH}$ guidelines.

The reduced run time and low cost of analysis are additional merits of the method. This method showed the best resolution by using pre-packed Nucleosil $\mathrm{C}_{18}(10 \mu \mathrm{m}, 25 \times 0.46 \mathrm{~cm})$ column at ambient temperature. The mobile phase consisting of methanol:water:acetonitrile (55:35:10 v/v; pH adjusted to 2.65 with phosphoric acid) was pumped at a flow rate of $1.0 \mathrm{~mL} \mathrm{~min}{ }^{-1}$ with an average operating pressure of $130 \mathrm{~kg} / \mathrm{cm}^{2}$ and effluent was monitored at $238 \mathrm{~nm}$. Linearity of the method in the concentration range $5-100 \mathrm{~g} \mathrm{~mL}^{-1}$ for prazosin and $10-600 \mathrm{\mu g} \mathrm{mL}^{-1}$ for calcium channel blockers showed good linear relationships for all the analytes $\left(R^{2}<0.9998\right)$. The LLOD values were 32.8, 30.6, 54.2, 29.9 and LLOQ were 99.4, 92.6, 164.2, $90.5 \mathrm{ng} \mathrm{mL}^{-1}$ for PRZ, AML, DIL and VER respectively, as per ICH guide lines acceptance criteria of $98-102 \%$.

The newly developed method has been successfully employed for studying the interactions between prazosin and ca-channel blockers at simulated human body conditions; the results envisage a positive interaction between the two classes of drugs, as the percent recovery of the drugs almost changed, which indicate that prazosin may not be safe to co-administer with these antihypertensive drugs (Graphical Abstract).

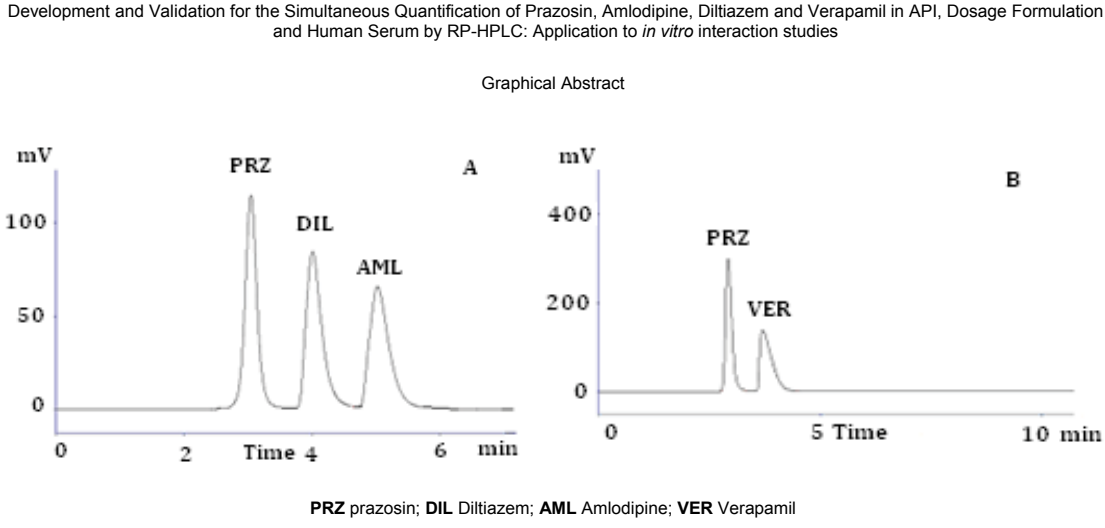

Keywords: Prazosin; Amlodipine; Diltiazem; Verapamil; Human serum; Interaction studies

\section{Introduction}

Prazosin hydrochloride (PRZ) a 1-(4-Amino-6,7-dimethoxy-2quinazolinyl)-4-(2-furanylcarbonyl) piperazine (Figure 1a) [1] belongs to class of $\alpha-1$ adrenergic blockers, which lowers the blood pressure by relaxing blood vessels. The central $\alpha_{1}$-adrenergic mechanism is an important component in the regulation of hypothalamic corticotropinreleasing hormone secretion during stress [2] and effects on behavioral alteration induced by MK-801 in a spatial memory task $[3,4]$ and has favorable effects on the plasma lipids, with reductions in total serum cholesterol [5]. PRZ can be used alone or in combination with thiazide diuretic and $\beta$ - blocker in the treatment of hypertension [6] and can also be administered in chronic heart failure with ACE - inhibitors [7-9]. PRZ and CCBs are given in combination in the treatment of essential hypertension [10]. Boman et al. [11] have suggested that addition of PRZ may be of value in patients with severe heart failure unresponsive to diuretics and digitalis combined with an ACE inhibitor, the combination of PRZ prazosin and an ACE inhibitor should theoretically reduce any activation of the rennin - angiotensin aldosterone system by prazosin.

*Corresponding author: Saeed Arayne M, Department of Chemistry, University of
Karachi, Karachi-75270, Pakistan, Tel: 922134664402; E-mail: msarayne@gmail.com Received October 22, 2014; Accepted December 01, 2014; Published December 03, 2014

Citation: Sultana N, Saeed Arayne M, Shah SN (2014) Development and Validation for the Simultaneous Quantification of Prazosin, Amlodipine, Diltiazem and Verapamil in API, Dosage Formulation and Human Serum by RP-HPLC Application to in vitro Interaction Studies. Med chem 4: 770-777. doi:10.4172/2161 0444.1000228

Copyright: $\odot 2014$ Sultana N, et al. This is an open-access article distributed under the terms of the Creative Commons Attribution License, which permits unrestricted use, distribution, and reproduction in any medium, provided the original author and source are credited. 
<smiles>COc1cc2nc(N3CCN(C(=O)c4ccco4)CC3)nc(N)c2cc1OC</smiles>

(a) Prazosin

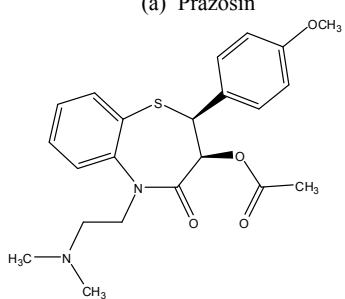

(c) Diltiazem

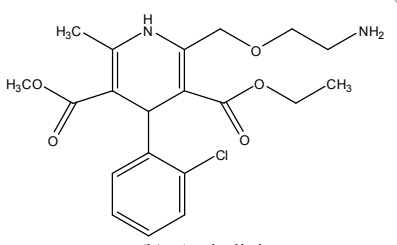

(b) Amlodipine

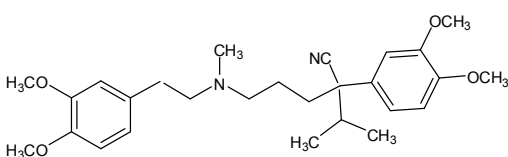

(d) Verapamil

Figure 1: Chemical structures of prazosin and CCBs.

Calcium channel blockers (CCBs) are a structurally and functionally heterogeneous group of medications that are used widely to control blood pressure and manage symptoms of angina [12]. CCBs are particularly effective against large vessel stiffness, one of the common causes of elevated systolic blood pressure in elderly patients [13]. Amlodipine is dihydropyridine calcium channel blocker, often used to reduce systemic vascular resistance and arterial pressure, but are not used to treat angina, which carry an indication to treat chronic stable angina as well as vasospastic angina) because the vasodilation and hypotension can lead to reflex tachycardia. It can worsen proteinuria in patients with nephropathy [14]. Verapamil is phenylalkylamine calcium channel blocker, derived from phenethylamine, is relatively selective for myocardium, reduce myocardial oxygen demand and reverse coronary vasospasm, and is often used to treat angina. It has minimal vasodilatory effects compared with dihydropyridines and therefore causes less reflex tachycardia, which can be the most significant contributor to the heart's need for oxygen making it appealing for treatment of angina. Therefore, as vasodilation is minimal with the phenylalkylamines, the major mechanism of action is causing negative inotropy [15]. Caution should be taken when using verapamil with a $\beta$-blocker due to the risk of severe bradycardia [16]. Diltiazem a benzothiazepine calcium channel blocker belongs to the benzothiazepine class of compounds and is an intermediate class between phenylalkylamine and dihydropyridines in its selectivity for vascular calcium channels. It is administered orally in the treatment of angina pectoris and hypertension and may be given intravenously in the treatment of arterial fibrillation or flutter and paroxysmal supraventricular tachycardia [17]. The structures of CCBs used in this study are given in Figures 1b-d.

Literature survey has reported a number of analytical techniques for the determination of prazosin in biological fluids and pharmaceutical formulations by potentiometric titration [18], high performance liquid chromatography (HPLC) [19] and with fluorescence detector [20]. We have reported the quantitation of prazosin in active pharmaceutical ingredients (API), dosage formulations and serum and the method has been applied to study its interaction with metals [21]. Sahu and Patel [22] and Akram and El-Didamony [23] have reported the quantification of diltiazem, amlodipine and verapamil by ultraviolet (UV) spectrophotometry. High-performance liquid chromatographic (HPLC) method for the determination of diltiazem has been reported by Sultana et al. [24], whereas other workers have reported simultaneous determination of these Ca-channel blockers with other co-administered drugs [25-27]. Our research group earlier reported methods for the

\begin{tabular}{|c|c|c|c|}
\hline Brand & Active Material & Drug $(\mathbf{m g})$ per tablet & Manufacturer \\
\hline Minipress & Prazosin & $2 \mathrm{mg}$ & Pfizer Pakistan Ltd \\
\hline Norvasc & Amlodipine & $5 \mathrm{mg}$ & Pfizer Pakistan Ltd \\
\hline Dilzem & Diltiazem & $30 \mathrm{mg}$ & Park-Davis \& Company Ltd \\
\hline Calan & Verapamil & $40 \mathrm{mg}$ & Searle Pakistan Ltd \\
\hline
\end{tabular}

Table 1: Details of API and dosage formulations

simultaneous determination of diltiazem and quinolones [28] and diltiazem with antidiabetic drugs [29] by HPLC.

Earlier we reported the simultaneous determination of prazosin with statins, in combined pharmaceutical dosage forms [30]. Literature reported that combination of CCBs and adrenergic receptor inhibitor could express more profound effects [31]. In present work, we developed a simultaneous HPLC method with UV detection for the quantitation of PRZ with AML, DIL and VER in commercial tablets and human serum, which has not been yet reported. The developed method is validated and has been used to study interactions between PRZ and CCBs (amlodipine, diltiazem and verapamil) for the routine monitoring of hypertensive patients who take a combination of these medications, and to study the pharmacokinetics of the combined dosage forms.

\section{Experimental}

\section{Material and methods}

The details API of prazosin and CCBs and their respective dosage forms are listed in Table 1. The API were kind gift from these manufacturers, while the dosage formulations were purchased from local pharmacies. They had an expiry of not less than one year at the time of study. All reagents used were of HPLC grade. Acetonitrile, methanol, and phosphoric acid 85\% (E. Merck, Darmstadt, Germany) and HPLC-grade deionized water and working solutions were prepared daily. All solutions were filtered through a $0.45 \mu \mathrm{m}$ membrane filter (Millipore, Bedford, MA, USA) and degassed using a sonicator (Elma Germany).

\section{Instrumentation}

Two Shimadzu HPLC systems both from Shimadzu Corporation, Kyoto, Japan, one equipped with a liquid chromatography (LC)-10 AT VP pump and an SPD-10 A VP ultraviolet-visible detector, and another equipped with an LC-20AT and SPD-20A UV-VIS detector were utilized. Both the chromatographic systems were integrated via Shimadzu model CBM-102 to independent Intel Pentium IV computer (Intel Corporation, Santa Clara, CA, USA) loaded with Shimadzu CLASS-VP software (version 5.03, Shimadzu Corporation Japan) for data acquisition. A rheodyne manual injector (model 7725; Chrom Tech, Inc, Apple Valley, MN, USA) was fitted with a $20 \mu \mathrm{L}$ loop, Nuclosil $100-10$ C-18 column ( $250 \mathrm{~mm} \times 4.6 \mathrm{~mm}, 10$ microns), and a DGU-14 AM online degasser (Shimadzu Corporation) were also used. In addition, electrical balance (Mettler Toledo \# AB54), pH meter (Mettler Toledo MP 220), UV-Visible 1601 Shimadzu double beam spectrophotometer, $1 \mathrm{~cm}$ rectangular quartz cells, microliter syringe and micropore filtration assembly were used in this study.

\section{Chromatographic conditions}

The analysis was carried out isocratically with a mobile phase consisting of methanol:water:acetonitrile $(55: 35: 10 \mathrm{v} / \mathrm{v} / \mathrm{v})$, with the $\mathrm{pH}$ adjusted to $2.65 \pm 0.02$ using phosphoric acid (85\%). The sample was injected through a $20 \mu \mathrm{L}$ loop and the mobile phase after filtration 
Citation: Sultana N, Saeed Arayne M, Shah SN (2014) Development and Validation for the Simultaneous Quantification of Prazosin, Amlodipine, Diltiazem and Verapamil in API, Dosage Formulation and Human Serum by RP-HPLC: Application to in vitro Interaction Studies. Med chem 4: 770-777. doi:10.4172/2161-0444.1000228

through a $0.45 \mu \mathrm{m}$ membrane filter was pumped at a flow rate of $1 \mathrm{ml}$ $\mathrm{min}^{-1}$. The effluent was monitored at $238 \mathrm{~nm}$ and analyses were carried out at ambient temperature $\left(\sim 25^{\circ} \mathrm{C}\right)$.

\section{Preparation of standard and working solutions}

Primary solutions of PRZ, AML, DIL \& VER reference standards in $100,300,200$ and $600 \mu \mathrm{g} \mathrm{ml}^{-1}$ concentrations were prepared in a mixture of methanol and water (80:20) and appropriately diluted in concentration range of 5-100 $\mu \mathrm{g} \mathrm{mL}$ of PRZ, 10-200 $\mu \mathrm{g} \mathrm{mL}$ of DIL, 15-300 $\mu \mathrm{g} \mathrm{mL}$ of AML, and 30-600 $\mu \mathrm{g} \mathrm{mL}$ of VER in the same solvent. These solutions were then injected into the LC system $(n=6)$.

Pharmaceutical formulations: 20 tablets of each pharmaceutical formulation (Table 1) were accurately weighed and finely powdered. Amount of powder equivalent to $10 \mathrm{mg}$ of PRZ, $20 \mathrm{mg}$ of DIL, 30 $\mathrm{mg}$ of AML and $60 \mathrm{mg}$ of VER were transferred individually to 100 $\mathrm{mL}$ volumetric flasks and dissolved by sonication with intermittent intervals for 60 minutes in methanol:water (80:20). Each flask was filled to mark and the resulting solutions were filtered through $0.45 \mu \mathrm{m}$ filter. The aliquots were further diluted to get the desired concentrations.

Sample preparation for serum: Blood samples were collected from healthy volunteers (who consent was already sought and none of those were on medication, smoking or in any other strenuous activity) in evacuated glass tubes through an indwelling cannula placed on forearm vein by a trained clinical laboratory technician (Fatmid Foundation Hospital, Karachi, Pakistan). The blood was shaken with acetonitrile

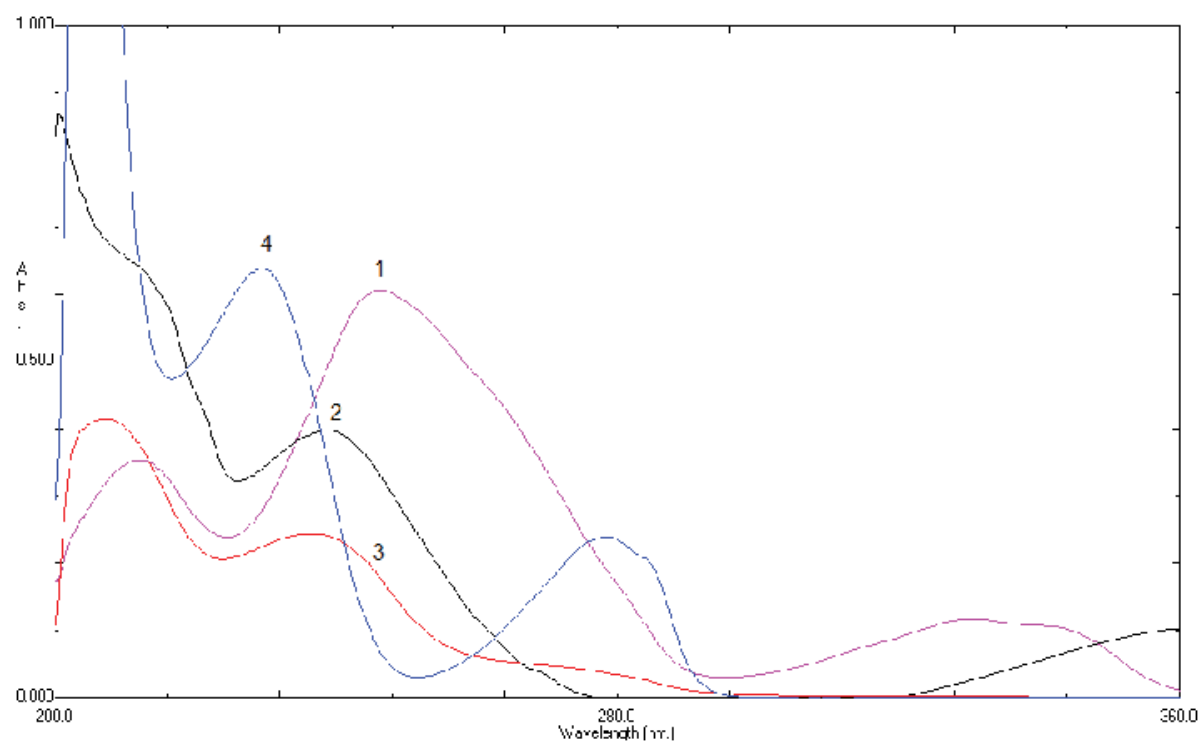

Figure 2: UV spectrum of 1=prazosin, 2=amlodipine, 3=diltiazem and 4=verapamil.
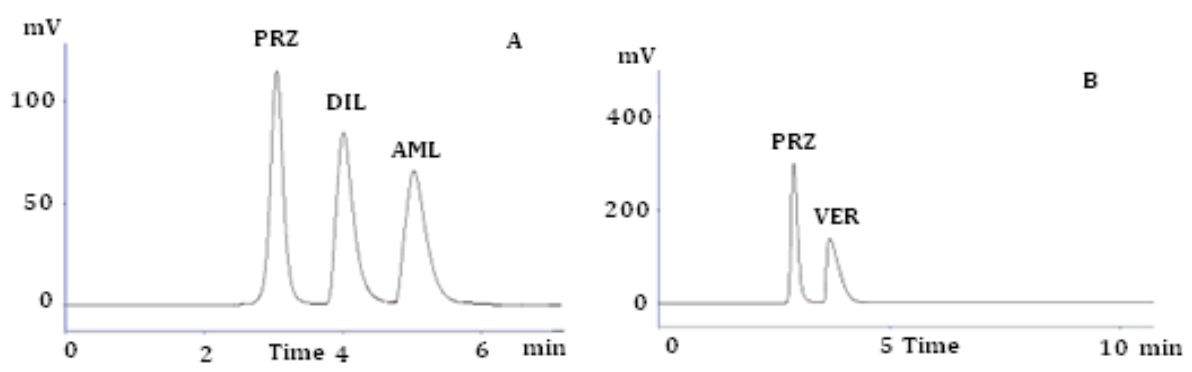

Figure 3: Chromatogram of prazosin, diltiazem and amlodipine (A); prazosin and verapamil (B) in formulation.
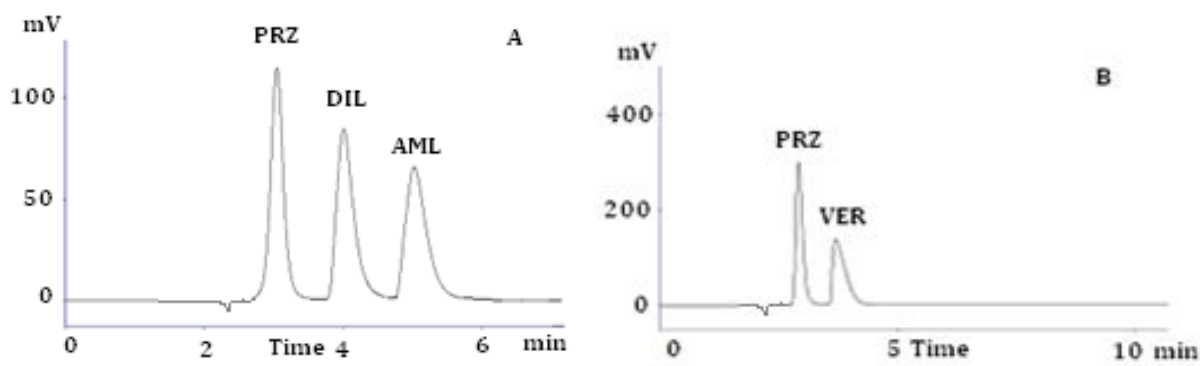

Figure 4: Chromatogram of prazosin, diltiazem and amlodipine (A); prazosin and verapamil $(B)$ in human serum 
Citation: Sultana N, Saeed Arayne M, Shah SN (2014) Development and Validation for the Simultaneous Quantification of Prazosin, Amlodipine, Diltiazem and Verapamil in API, Dosage Formulation and Human Serum by RP-HPLC: Application to in vitro Interaction Studies. Med chem 4: 770-777. doi:10.4172/2161-0444.1000228

\begin{tabular}{|c|c|c|c|c|c|}
\hline Drugs & Concentration ( $\left.\mu \mathrm{g} \mathrm{mL}^{-1}\right)$ & Regression equation & $r^{2}$ & LLOD $\mathrm{ng} \mathrm{mL}^{-1}$ & LLOQ $\mathrm{ng} \mathrm{mL}^{-1}$ \\
\hline Prazosine & $5-100$ & $y=29490 x+29699$ & 0.9999 & 32.8 & 99.4 \\
\hline Amlodipine & $15-300$ & $y=27735 x+18635$ & 0.9998 & 30.6 & 92.6 \\
\hline Diltiazem & $10-200$ & $y=29072 x+17699$ & 0.9999 & 54.2 & 164.2 \\
\hline Verapamil & $30-600$ & $y=26185 x+39843$ & 0.9999 & 29.9 & 90.5 \\
\hline
\end{tabular}

Table 2: Regression parameters

\begin{tabular}{|c|c|c|c|c|c|}
\hline \multirow{2}{*}{ Analyte } & \multirow{2}{*}{$\begin{array}{l}\text { Spiked conc. } \\
\left(\mu \mathrm{g} \mathrm{mL}^{-1}\right)\end{array}$} & \multicolumn{2}{|c|}{ In bulk } & \multicolumn{2}{|c|}{ In serum } \\
\hline & & \% Rec. & $\%$ RSD & \% Rec. & $\%$ RSD \\
\hline \multirow{3}{*}{ Prazosin } & 8 & 100.08 & 0.009 & 100.04 & 0.004 \\
\hline & 10 & 99.93 & 0.004 & 99.97 & 0.002 \\
\hline & 12 & 100.65 & 0.001 & 100.07 & 0.008 \\
\hline \multirow{3}{*}{ Amlodipine } & 32 & 100.21 & 0.008 & 100 & 0.018 \\
\hline & 40 & 100.09 & 0.085 & 100.03 & 0.008 \\
\hline & 48 & 99.55 & 0.002 & 99.98 & 0.001 \\
\hline \multirow{3}{*}{ Diltiazem } & 16 & 100.17 & 0.007 & 100.06 & 0.011 \\
\hline & 20 & 100.09 & 0.005 & 99.96 & 0.005 \\
\hline & 24 & 100.1 & 0.001 & 100.03 & 0.002 \\
\hline \multirow{3}{*}{ Verapamil } & 48 & 100.05 & 0.002 & 100.05 & 0.004 \\
\hline & 60 & 99.94 & 0.003 & 99.92 & 0.004 \\
\hline & 72 & 99.98 & 0.054 & 100.09 & 0.017 \\
\hline
\end{tabular}

Table 3: Accuracy of the proposed method

\begin{tabular}{|c|c|c|c|c|c|c|c|c|c|c|c|c|}
\hline \multirow{2}{*}{ Conc. $\mu \mathrm{g} \mathrm{mL}^{-1}$} & \multicolumn{4}{|c|}{ Day 1 (\% Recovery) } & \multicolumn{4}{|c|}{ Day 2 (\% Recovery) } & \multicolumn{4}{|c|}{ Day 3 (\% Recovery) } \\
\hline & PRZ & AML & DIL & VER & PRZ & AML & DIL & VER & PRZ & AML & DIL & VER \\
\hline \multicolumn{13}{|l|}{ API } \\
\hline 5 & 99.28 & 99.79 & 100.2 & 100.0 & 99.98 & 99.25 & 101.2 & 100.5 & 99.52 & 99.82 & 100.5 & 100.3 \\
\hline 10 & 99.93 & 100.1 & 100.1 & 99.94 & 99.85 & 98.99 & 100.2 & 99.24 & 99.74 & 99.51 & 99.58 & 98.83 \\
\hline 15 & 98.95 & 100.0 & 99.81 & 99.58 & 100.2 & 98.74 & 100.5 & 100.7 & 99.94 & 99.29 & 100.1 & 100.2 \\
\hline 20 & 99.71 & 99.60 & 98.59 & 99.90 & 99.65 & 99.68 & 99.87 & 99.83 & 99.28 & 100.7 & 99.58 & 99.57 \\
\hline 25 & 100.2 & 100.5 & 99.96 & 100.3 & 98.59 & 99.89 & 100.7 & 99.17 & 99.51 & 99.59 & 101.2 & 98.46 \\
\hline 50 & 100.7 & 99.28 & 100.2 & 100.6 & 100.2 & 100.1 & 100.0 & 100.5 & 100.0 & 99.87 & 99.51 & 100.1 \\
\hline 100 & 99.97 & 98.89 & 100.1 & 99.08 & 99.87 & 99.05 & 99.96 & 100.9 & 99.57 & 99.26 & 99.27 & 99.87 \\
\hline \multicolumn{13}{|l|}{ Serum } \\
\hline 5 & 100.0 & 99.28 & 99.86 & 99.95 & 100.2 & 99.16 & 100.8 & 100.2 & 99.01 & 99.51 & 100.2 & 100.1 \\
\hline 10 & 99.65 & 99.58 & 100.0 & 100.1 & 100.5 & 98.58 & 100.4 & 9.65 & 99.97 & 99.01 & 100.7 & 99.28 \\
\hline 15 & 99.18 & 99.71 & 99.67 & 100.8 & 100.8 & 98.11 & 100.2 & 100.4 & 98.28 & 99.64 & 99.96 & 99.17 \\
\hline 20 & 99.50 & 99.83 & 99.58 & 99.92 & 99.85 & 99.19 & 100.1 & 99.92 & 99.87 & 98.17 & 99.57 & 99.51 \\
\hline 25 & 99.68 & 100.1 & 100.0 & 100.2 & 99.69 & 99.38 & 100.9 & 99.75 & 100.2 & 99.84 & 101.0 & 100.1 \\
\hline 50 & 99.98 & 99.05 & 99.68 & 100.6 & 99.53 & 99.82 & 99.58 & 100.1 & 99.17 & 99.63 & 99.15 & 99.47 \\
\hline 100 & 99.37 & 99.18 & 101.0 & 99.87 & 99.08 & 98.95 & 100.2 & 100.4 & 98.96 & 99.50 & 100.0 & 99.96 \\
\hline
\end{tabular}

Table 4: Intra-and inter day, variation /intermediate precision of the method

\begin{tabular}{|c|c|c|c|c|c|}
\hline \multirow{2}{*}{$\begin{array}{c}\text { Parameters } \\
\text { Chromatographic Condition }\end{array}$} & \multirow[b]{2}{*}{ Variation } & \multicolumn{4}{|c|}{$\%$ RSD } \\
\hline & & PRZ & AML & DIL & VER \\
\hline \multirow{2}{*}{ Mobile phase composition } & $\mathrm{MeOH}: \mathrm{H}_{2} \mathrm{O}: \mathrm{ACN}(50: 40: 10)$ & 0.047 & 0.011 & 0.015 & 0.057 \\
\hline & $\mathrm{MeOH}: \mathrm{H}_{2} \mathrm{O}: \mathrm{CAN}(60: 30: 10)$ & 0.01 & 0.017 & 0.019 & 0.021 \\
\hline \multirow{2}{*}{$\mathrm{pH}$ of mobile phase } & 2.63 & 0.745 & 0.042 & 0.054 & 0.145 \\
\hline & 2.67 & 0.014 & 0.019 & 0.014 & 0.021 \\
\hline \multirow{2}{*}{ Flow rate $\left(\mathrm{mL} \mathrm{min}^{-1}\right)$} & 0.9 & 0.241 & 0.032 & 0.061 & 0.01 \\
\hline & 1.1 & 0.417 & 0.421 & 0.019 & 0.005 \\
\hline \multirow{2}{*}{ Column temperature $\left({ }^{\circ} \mathrm{C}\right)$} & 22 & 0.528 & 0.247 & 0.013 & 0.007 \\
\hline & 30 & 0.015 & 0.087 & 0.041 & 0.018 \\
\hline \multirow{2}{*}{ Detection wavelength $(\mathrm{nm})$} & 238 & 0.065 & 0.024 & 0.081 & 0.045 \\
\hline & 242 & 0.021 & 0.004 & 0.014 & 0.085 \\
\hline
\end{tabular}

Table 5: Robustness of the method

$(10: 90, \mathrm{v} / \mathrm{v})$ and centrifuged at $10,000 \mathrm{rpm}$ for $10 \mathrm{~min}$ to deprotonate it. The serum thus obtained was filtered and used for the analysis [32]. Stock standard solutions were spiked with serum to prepare desired working solutions and analyzed. These serum solutions were stored at $-20^{\circ} \mathrm{C}$ for pending analysis and for inter day variation studies.

\section{Sample preparation for interaction studies}

Stock solutions of PRZ, DIL, AML and VER in $100 \mu \mathrm{g} \mathrm{mL}{ }^{-1}$ concentration were individually prepared in buffers of $\mathrm{pH} 1,4,7.4$ and 9 to simulate human GI tract. PRZ was then mixed with each of calcium channel blockers separately in 1:1 ratio in Erlenmeyer flasks, which gave the final concentration of $50 \mu \mathrm{g} \mathrm{mL}^{-1}$ for each constituent. The flasks were maintained at $37^{\circ} \mathrm{C}$ for three hours with frequent shaking. Aliquots of $2 \mathrm{ml}$ were withdrawn at 30 minute intervals for 180 minutes, appropriately diluted, filtered (through Millipore filter $0.45 \mu \mathrm{M})$ and chromatographed. 
Citation: Sultana N, Saeed Arayne M, Shah SN (2014) Development and Validation for the Simultaneous Quantification of Prazosin, Amlodipine, Diltiazem and Verapamil in API, Dosage Formulation and Human Serum by RP-HPLC: Application to in vitro Interaction Studies. Med chem 4: 770-777. doi:10.4172/2161-0444.1000228

\begin{tabular}{|c|c|c|c|c|}
\hline \multirow{2}{*}{ Drugs } & \multirow{2}{*}{ System } & \multicolumn{2}{|c|}{ Concentration $\left(\mu \mathrm{g} \mathrm{mL}^{-1}\right)$} & \multirow{2}{*}{ Recovery (\%) } \\
\hline & & Added & Found & \\
\hline \multirow{6}{*}{ PRZ } & \multirow{3}{*}{ LC 10} & 8 & 8.01 & 100.08 \\
\hline & & 10 & 9.99 & 99.93 \\
\hline & & 12 & 12.08 & 100.65 \\
\hline & \multirow{3}{*}{ LC 20} & 8 & 7.99 & 99.85 \\
\hline & & 10 & 10 & 99.99 \\
\hline & & 12 & 12.1 & 100.85 \\
\hline \multirow{6}{*}{ AML } & \multirow{3}{*}{ LC 10} & 32 & 32.07 & 100.21 \\
\hline & & 40 & 40.04 & 100.09 \\
\hline & & 48 & 47.78 & 99.55 \\
\hline & \multirow{3}{*}{ LC 20} & 32 & 32.17 & 100.54 \\
\hline & & 40 & 40.44 & 101.1 \\
\hline & & 48 & 48 & 100.01 \\
\hline \multirow{6}{*}{ DIL } & \multirow{3}{*}{ LC 10} & 16 & 16.03 & 100.17 \\
\hline & & 20 & 20.02 & 100.09 \\
\hline & & 24 & 24.02 & 100.1 \\
\hline & \multirow{3}{*}{ LC 20} & 16 & 15.88 & 99.28 \\
\hline & & 20 & 20.05 & 100.26 \\
\hline & & 24 & 24.35 & 101.45 \\
\hline \multirow{6}{*}{ VER } & \multirow{3}{*}{ LC 10} & 48 & 48.02 & 100.05 \\
\hline & & 60 & 59.96 & 99.94 \\
\hline & & 72 & 71.99 & 99.98 \\
\hline & \multirow{3}{*}{ LC 20} & 48 & 48.67 & 101.4 \\
\hline & & 60 & 60.02 & 100.04 \\
\hline & & 72 & 72.34 & 100.47 \\
\hline
\end{tabular}

Table 6: Ruggedness of the proposed method

\section{Results and Discussion}

The method developed for the simultaneous quantitation of prazosin with calcium channel blockers is simple, precise, accurate and sensitive. Chromatographic conditions were optimized to achieve best separation and to get best resolution between analytes and to optimize chromatographic parameters like resolution, tailing factor and retention time. For validation of analytical methods, the ICH guidelines [33] and USP 2007 [34] were followed for the specificity, accuracy tests, precision, linearity, work strip and robustness of the method.

\section{Method development and optimization}

Some chromatographic parameters such as column type, mobile phase, wavelength and conditioning time were investigated to obtain a suitable peak for all analytes within an acceptable time and limit. In order to optimize column packing, $\mathrm{C}_{8}$ and $\mathrm{C}_{18}$ columns with different lengths and particle sizes were used. Nuclosil, $\mathrm{C}_{18}(250 \times 4.6 \mathrm{~mm}, 10 \mu \mathrm{m})$ column provided an efficient and reproducible peak area with sharp peaks at ambient temperature. Different combinations and ratios of methanol, water and acetonitrile were tried for mobile phase but the best composition, which delivered optimum results was achieved with methanol, water and acetonitrile in the ratio of 55:35:10 (v/v/v). However, the $\mathrm{pH}$ of this mobile phase was to be adjusted to $2.65 \pm$ 0.02 with phosphoric acid to have good resolution without tailing or fronting. Variation in $\mathrm{pH}$ of the mobile phase also had a great influence to the retention time and peak sharpness of analytes. If percentage of methanol in the mobile phase was increased the retention time of CCBs decreased and the peaks merged with prazosin. The optimum wavelength selected for analysis was on the basis of UV scan of all interacting drugs, which showed maximal absorbance near $238 \mathrm{~nm}$ (Figure 2).

System suitability testing: An integral part of chromatographic methods, system suitability test was used to verify the resolution and
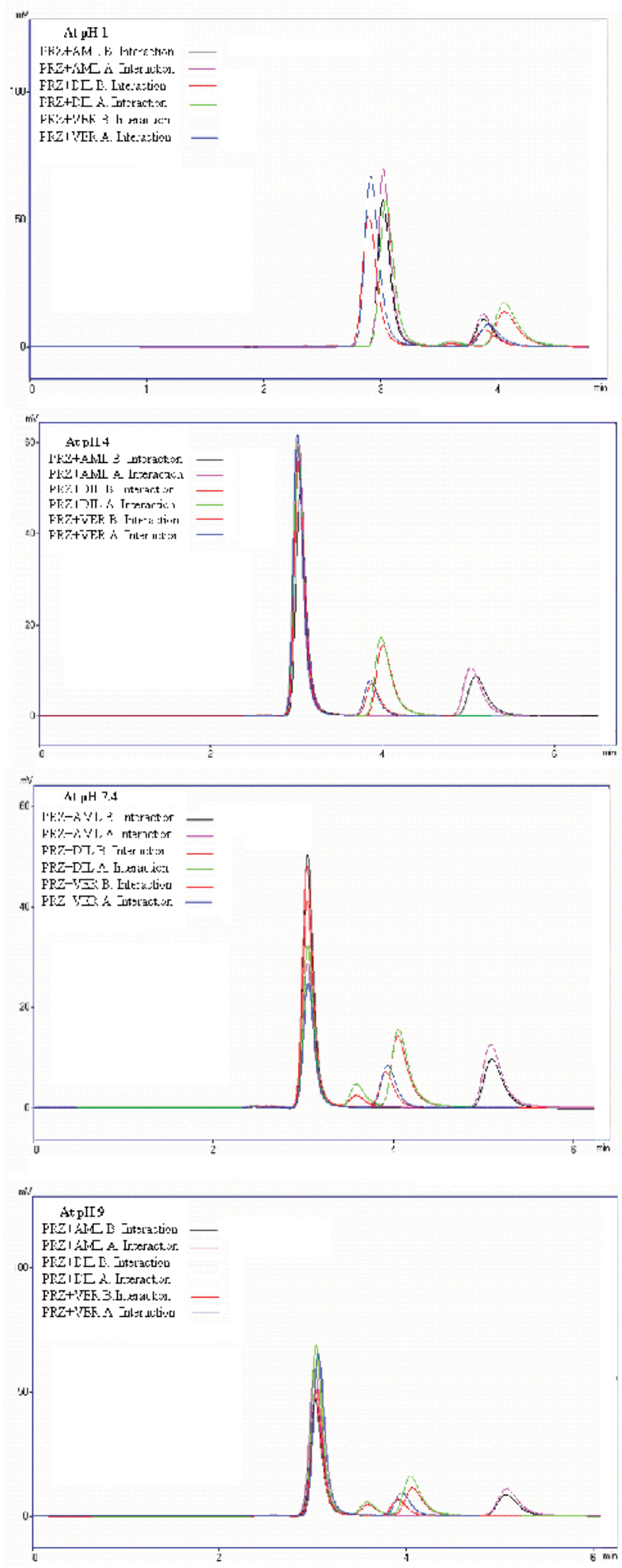

Figure 5: Chromatograms showing drift in retention time as well as change in $\mathrm{AUC}$ of drugs, at various $\mathrm{pH}$ levels. $\mathrm{B}=$ before interaction; $\mathrm{A}=$ after interaction.

reproducibility of the system for adequate analysis. The HPLC system was equilibrated with the mobile phase, followed by six injections of the standard. These consecutive injections were used to evaluate the system suitability on each day of method validation. All peaks were 
Citation: Sultana N, Saeed Arayne M, Shah SN (2014) Development and Validation for the Simultaneous Quantification of Prazosin, Amlodipine, Diltiazem and Verapamil in API, Dosage Formulation and Human Serum by RP-HPLC: Application to in vitro Interaction Studies. Med chem 4: 770-777. doi:10.4172/2161-0444.1000228

well resolved and the precision was acceptable. Parameter of system suitability was peak symmetry (symmetry factor), theoretical plates of the column, mass distribution ratio (capacity factor) and tailing factor.

Specificity and selectivity: Selectivity and specificity of the chromatographic method was determined to ensure the separation of prazosin and CCBs. The method demonstrated fine resolutions and was found to be free of interference from excipients (Figure 3) used in dosage formulations; thus the method was specific for prazosin and CCBs (Figure 4) in serum as well. These chromatograms showed no additional peaks due to excipients or endogenous serum components at the same retention time of analyte, and hence the specificity was confirmed.

Range and linearity: Linearity is generally reported as the variance of the slope of the regression line. It was tested in order to demonstrate a proportional relationship of response verses analyte concentration. It was studied at seven concentration levels in the range of 5-100 $\mu \mathrm{g}$ $\mathrm{mL}^{-1}$ for prazosin; $15-300 \mu \mathrm{g} \mathrm{mL}^{-1}$ for amlodopine, $10-200 \mu \mathrm{g} \mathrm{mL}^{-1}$ for diltiazem and $30-600 \mu \mathrm{g} \mathrm{mL} \mathrm{m}^{-1}$ for verapamil. Six replicates were performed for every concentration. Injected concentrations versus peak area were plotted, and the correlation coefficients were calculated, which are given in Table 2 .

Accuracy and recovery studies: The accuracy of the procedure which gauge the closeness of measured values to the true values was evaluated as percentage relative error between the measured mean concentrations and taken concentrations. A minimum of 3 concentration levels covering the specified ranges were selected and six runs were performed for every concentration and then \% recovery was calculated (Table 3).

Precision: The intra- and inter-day variability or precision was evaluated by assaying the sample's seven concentrations per day for three consecutive days (Table 4). In this assay, the intra-day precision and the inter-day precision recovery was $98-102 \%$. The $\%$ RSD was found to be less than $2 \%$, which indicated that the method was sufficiently precise and accurate.

Limits of detection and quantitation: The ratio of chromatographic signal when remained three times and ten times with their respective noise signals was concluded for LLOD and LLOQ respectively. The LLOD and LLOQ for PRZ, AML, DIL and VER were 32.8, 30.6, 54.2 and $29.9 \mathrm{ng} \mathrm{mL}^{-1}$ and $99.4,92.6,164.2$ and $90.5 \mathrm{ng} \mathrm{mL}^{-1}$ respectively in bulk material (Table 2).

Robustness: Robustness of the method was accomplished by design modifications made to the method parameters such as composition of mobile phase, flow rate, $\mathrm{pH}$ of the mobile phase, detection wavelength, and column temperature (Table 5), and it was found that the percentage of RSD values did not exceed more than $2 \%$.

Ruggedness: The ruggedness of the study was established by determining PRZ, AML, DIL, and VER using two different chromatographic systems LC10 and LC20 and same column. The assay results indicated that the method was equally capable with high precision (Table 6) on both the instruments.

\begin{tabular}{|c|c|c|c|c|c|c|c|c|}
\hline \multirow{3}{*}{$\begin{array}{c}\text { Time } \\
\text { (mins) }\end{array}$} & \multicolumn{2}{|c|}{ pH 1} & \multicolumn{2}{|c|}{ pH 4} & \multicolumn{2}{|c|}{ pH 7.4} & \multicolumn{2}{|c|}{ pH 9} \\
\hline & \multicolumn{8}{|c|}{ Prazosin and amlodipine (available \%) } \\
\hline & PRZ & AML & PRZ & AML & PRZ & AML & PRZ & AML \\
\hline 0 & 99.89 & 98.78 & 99.59 & 99.48 & 100.15 & 100.28 & 100.29 & 98.48 \\
\hline 30 & 92.19 & 93.72 & 100.19 & 99.12 & 105.00 & 102.93 & 104.58 & 97.74 \\
\hline 60 & 93.75 & 95.30 & 107.24 & 106.57 & 104.90 & 104.97 & 100.77 & 101.99 \\
\hline 90 & 98.67 & 100.34 & 110.08 & 109.81 & 79.35 & 107.29 & 110.85 & 111.23 \\
\hline 120 & 108.21 & 110.79 & 114.40 & 110.73 & 62.70 & 112.19 & 118.26 & 119.53 \\
\hline 150 & 114.94 & 116.23 & 116.43 & 110.40 & 63.61 & 120.26 & 121.90 & 119.07 \\
\hline 180 & 119.59 & 122.08 & 119.40 & 117.34 & 55.44 & 127.20 & 131.78 & 130.38 \\
\hline \multicolumn{9}{|c|}{ Prazosin and Diltiazem (available \%) } \\
\hline & PRZ & DIL & PRZ & DIL & PRZ & DIL & PRZ & DIL \\
\hline 0 & 101.21 & 100.85 & 100.10 & 99.98 & 100.12 & 100.25 & 100.28 & 100.59 \\
\hline 30 & 104.26 & 105.04 & 101.59 & 100.38 & 104.55 & 104.09 & 101.12 & 101.79 \\
\hline 60 & 107.37 & 107.07 & 101.97 & 101.58 & 77.84 & 106.42 & 100.43 & 98.47 \\
\hline 90 & 108.84 & 108.91 & 100.70 & 101.79 & 79.79 & 106.38 & 111.24 & 113.06 \\
\hline 120 & 109.99 & 109.94 & 101.51 & 102.33 & 48.75 & 106.33 & 117.43 & 124.39 \\
\hline 150 & 114.86 & 115.81 & 110.14 & 109.98 & 52.74 & 106.41 & 119.63 & 123.68 \\
\hline 180 & 116.93 & 117.08 & 110.68 & 110.76 & 61.24 & 117.30 & 133.12 & 141.07 \\
\hline \multicolumn{9}{|c|}{ Prazosin and Verapamil (available \%) } \\
\hline & PRZ & VER & PRZ & VER & PRZ & VER & PRZ & VER \\
\hline 0 & 100.54 & 101.12 & 99.89 & 100.21 & 99.87 & 99.78 & 100.01 & 100.12 \\
\hline 30 & 122.85 & 115.74 & 99.99 & 101.01 & 98.54 & 99.42 & 102.57 & 102.72 \\
\hline 60 & 120.98 & 112.95 & 102.25 & 103.09 & 96.63 & 101.47 & 108.06 & 105.06 \\
\hline 90 & 129.20 & 120.11 & 102.67 & 103.74 & 77.00 & 102.85 & 115.62 & 115.91 \\
\hline 120 & 139.38 & 131.47 & 107.34 & 109.61 & 61.78 & 107.80 & 117.44 & 117.14 \\
\hline 150 & 145.90 & 134.07 & 107.61 & 108.47 & 61.06 & 109.65 & 130.29 & 131.88 \\
\hline 180 & 158.10 & 150.22 & 108.61 & 111.03 & 50.86 & 117.27 & 142.63 & 143.29 \\
\hline
\end{tabular}

Table 7: Interaction studies between prazosin and calcium channel blockers at $37^{\circ} \mathrm{C}$ 
Citation: Sultana N, Saeed Arayne M, Shah SN (2014) Development and Validation for the Simultaneous Quantification of Prazosin, Amlodipine, Diltiazem and Verapamil in API, Dosage Formulation and Human Serum by RP-HPLC: Application to in vitro Interaction Studies. Med chem 4: 770-777. doi:10.4172/2161-0444.1000228

\section{Interaction studies using RP-HPLC}

Results of interactions studies of prazosin with calcium channel blockers are summarized in Table 7. The recovery of PRZ increased to $120--132 \%$ in presence of AML in simulated gastric juice, buffers of $\mathrm{pH}$ 4 and 9, while in buffer of $\mathrm{pH} 7.4$ the recovery of PRZ first increased to $105 \%$ and later decreased to $55 \%$ at the end of the experiment. On the other hand, the recovery of AML increased up to $122-130 \%$ in all interacting mediums. In case of both DIL and VER the recovery of prazosin increased in all buffers, whereas the recovery of $\mathrm{PRZ}$ in $\mathrm{pH}$ 7.4 decreased to 61 and $51 \%$ respectively at the end of the experiment. Similar behavior was observed in case of VER, the recovery increased in all buffers. Conclusively, a remarkable change in AUC and drift in retention time (Figure 5) are evident of the interactions of PRZ with CCBs under study. Hence, it would not be safe to co-administer prazosin with these interacting drugs. These two classes of drugs inhibit or disturb the absorption of each other.

\section{Conclusion}

High performance liquid chromatographic method for the simultaneous quantitation of prazosin and calcium channel blockers (amlodipine, diltiazem and verapamil) was developed and validated for the first time. The current method is very simple, fast and easy to perform, shows a high percentage of precision and recoveries, shorter run time, has low limits of detection and quantification values up to nanogram levels and is linear up to a wide range of concentrations. This makes the method applicable for routine clinical analysis, multidrug pharmacokinetic study and also in the study of drug- drug interactions.

The developed method has been applied to the monitoring of in vitro interactions of PRZ with these CCBs. In the light of above results, it is inferred that certain medicines can interact pharmacologically and affect the activity of others if they are mixed during their administration. Hence the concurrent administration of these drugs may affect the bioavailability of PRZ, which may result in synergistic or antagonistic therapeutic effect.

\section{References}

1. Maryadele J, O'Neil, Patricia E, Heckelman, Cherie B, Koch, Kristin J Roman (2006) The Merck Index: An encyclopedia of Chemicals, Drugs, and Biologicals. Fourteenth Edition, Merck \& Co., Inc., Whitehouse Station, NJ, USA

2. Kiss A, Aguilera G (1992) Participation of alpha 1-adrenergic receptors in the secretion of hypothalamic corticotropin-releasing hormone during stress. Neuroendocrinology 56: 153-160.

3. Stuchlik A, Petrasek T, Vales K (2009) Effect of alpha(1)-adrenergic antagonist prazosin on behavioral alterations induced by MK-801 in a spatial memory task in Long-Evans rats. Physiol Res 58: 733-740.

4. Bubenakova-Valesova V, Horacek J, Vrajova M, Hoschl C (2008) Models of schizophrenia in humans and animals based on inhibition of NMDA receptors. Neurosci Biobehav Rev 32: 1014-1023.

5. Graham RM (1989) Selective alpha1-adrenergic blockade, lipids, and coronary heart disease risk. Am J Med 87: 53S-56S.

6. Seedat YK, Seedat MA, Bhoola R (1977) Prazosin combined with thiazide diuretic and beta-blocker in the treatment of hypertension. Curr Med Res Opin 4: 627-629.

7. Shen H (2007) Illustrated Pharmacology Memory Cards: PharMnemonics. Minireview,13.

8. Ajayi AA, Afolabi MA, Balogun MO, Adigun AQ, Ajayi OE, et al. (1996) Ora therapy with combined enalapril, prazosin and hydrochlorothiazide in the acute treatment of severe hypertension in Nigerians. Eur J Clin Pharmacol 51: 45-48.

9. Sharpe DN, Coxon R (1982) Prazosin and captopril in chronic heart failure: comparison of acute haemodynamic and hormonal effects. Aust N Z J Med

\section{2: $483-488$.}

10. Elliott HL, Meredith PA, Campbell L, Reid JL (1988) The combination of prazosin and verapamil in the treatment of essential hypertension. Clin Pharmacol Ther 43: 554-560.

11. Boman K, Eriksson P, Slunga $L$ (1989) Is addition of prazosin beneficial in chronic heart failure refractory to angiotensin converting enzyme inhibition? Eur J Clin Pharmacol 37: 431-432.

12. Eisenberg MJ, Brox A, Bestawros AN (2004) Calcium channel blockers: an update. Am J Med 116: 35-43.

13. Nelson M (2010) Drug treatment of elevated blood pressure. Australian Prescriber 33: 108-112.

14. Remuzzi G, Schieppati A, Ruggenenti P (2002) Clinical practice. Nephropathy in patients with type 2 diabetes. N Engl J Med 346: 1145-1151.

15. Hockerman GH, Peterson BZ, Johnson BD, Catterall WA (1997) Molecular determinants of drug binding and action on L-type calcium channels. Annu Rev Pharmacol Toxicol 37: 361-396

16. Buckley N, Dawson A, Whyte I (2007) Calcium channel blockers. Medicine 35 599-602.

17. Martindale (2005) The Complete Drug of Reference, 34 th Edition, Pharmaceutical Press of Great Britian.

18. British Pharmacopoeia (2012) Volume I \& II, Monographs: Medicinal and Pharmaceutical Substances (Ph. Eur. Monograph 0856).

19. Ahmed FM, Shetty ASKR, Ahmed M, Sridhar BK (2010) RP-HPLC method for estimation of prazosin hydrochloride in pharmaceutical dosage form. Int $J$ Chem Sci 8: 1956-1964.

20. Twomey TM, Hobbs DC (1978) Analysis of prazosin in plasma by a sensitive high-performance liquid chromatographic-fluorescence method. J Pharm Sci 67: 1468-1469.

21. Sultana N, Arayne MS, Shah SN (2013) Liquid Chromatographic Analysis of Prazosin in API, Dosage Form and Serum: Application to Drug-Metal Interaction Studies. J Chromatograph Separat Techniq 4:197.

22. Sahu R, Patel VB (2007) Simultaneous spectrophotometric determination of amlodipine besylate and atorvastatin calcium in binary mixture. Indian $\mathrm{J}$ Pharmaceutical Sci 69: 110-111.

23. El-Didamony AM (2005) Indirect spectrophotometric determination of diltiazem hydrochloride in pure form and pharmaceutical formulations. Central European Journal of Chemistry 3: 520-536.

24. Sultana N, Arayne MS, Shafi N (2007) A validated method for the analysis of diltiazem in raw materials and pharmaceutical formulations by rp-HPLC. Pak $J$ Pharm Sci 20: 284-290.

25. Mustafa Ç, Kaynak MS, Altinöz S, Sahin S (2008) Validated HPLC Method Development:The Simultaneous Analysis of Amlodipine and Valsartan in Samples for Liver Perfusion Studies. Hacettepe Üniversitesi Eczacılık Fakültesi dergisi 28: 15-30.

26. Valentina A, Lavinia H, Mircioiu I, Monica SR, Cristina R, et al. (2008) Development and validation of a HPLC method for the simultaneous determination of Diltiazem and its active metabolite desacetyldiltiazem in human Plasma. In 13th Congress of Balkan Military Medical Committee 141.

27. Dorota K (2005) Simultaneous High-Performance Thin-Layer Chromatography Densitometric Assay of Trandolapril and Verapamil in the Combination Preparation. Journal of AOAC International 88: 1525-1529.

28. Sultana N, Arayne MS, Shah SN, Shafi N, Naveed S (2010) Simultaneous Determination of Prazosin, Atorvastatin, Rosuvastatin and Simvastatin in API, Dosage Formulations and Human Serum by RP-HPLC. Journal of the Chinese Chemical Society 57:1286-1292.

29. Sultana N, Arayne MS, Shafi N, Siddiqui FA, Hussain A (2011) Development and validation of new assay method for the simultaneous analysis of diltiazem, metformin, pioglitazone and rosiglitazone by RP-HPLC and its applications in pharmaceuticals and human serum. J Chromatogr Sci 49: 774-779.

30. Sultana N, Arayne MS, Nighat S, Naz A, Shah S N, et al. (2009) A RP-HPLC method for the simultaneous determination of diltiazem and quinolones in bulk, formulations and human serum. Journal of Chilian Chemical Society 54: 358-362.

31. Mills TA, Kawji MM, Cataldo VD, Pappas ND, O'Meallie LP, et al. (2004) Profound sinus bradycardia due to diltiazem, verapamil, and/or beta-adrenergic blocking drugs. J La State Med Soc 156: 327-331.

32. Venkatesh G, Ramanathan S, Mansor SM, Nair NK, Sattar MA, et al. (2007) Development and validation of RP-HPLC-UV method for simultaneous determination of buparvaquone, atenolol, propranolol, quinidine and verapamil: 
Citation: Sultana N, Saeed Arayne M, Shah SN (2014) Development and Validation for the Simultaneous Quantification of Prazosin, Amlodipine, Diltiazem and Verapamil in API, Dosage Formulation and Human Serum by RP-HPLC: Application to in vitro Interaction Studies. Med chem 4: 770-777. doi:10.4172/2161-0444.1000228

a tool for the standardization of rat in situ intestinal permeability studies. J Pharm Biomed Anal 43: 1546-1551.

33. The United States Pharmacopeia Asian Edition (2007) United States Pharmacopeial Convention, Inc., Rockville, MD.
34. ICH-Q2B Guideline for Industry (2005) Q2B Validation of Analytical Procedure: Methodology. International Conference on Harmonisation, Food and Drug Administration, U.S. Department of Health and Human Services. 